\title{
Readmission rates are associated with differences in the process of care in acute asthma
}

\author{
R Slack, C E Bucknall
}

\begin{abstract}
Objective-To test the hypothesis that sustained differences in readmission rate for acute asthma were associated with variations in clinical practice.

Design-Data were collected by retrospective review of case notes, using the criteria recomended by the British Thoracic Society.

Setting-Two city National Health Service (NHS) hospitals that had recorded a sustained difference in readmission rate for acute asthma.

Subjects-A random sample of 50 from each hospital, selected from all 16-44 year old patients discharged in 1992 with acute asthma (ninth revision of the international classification of diseases (ICD-9) 493).

Results-Hospital A had a lower readmission rate than hospital $B$. The sample groups were similar for age, sex, deprivation of area of residence, and severity of episode. Systemic corticosteroids were given early more often $(p=0.02)$ and oral corticosteroids were prescribed at discharge more often $(p=0.04)$ in hospital $A$. When a short course of oral corticosteroids was prescribed the duration stated was longer $(p=0.02)$ and inhaled corticosteroids were started or the dose increased more often $(p=0.02)$ in hospital $A$.

Conclusions-These results support the hypothesis that differences in readmission rates for acute asthma are associated with variations in clinical practice. Sustained variation in readmission rates is an outcome of health care, for acute asthma. The findings also support audit of the process of hospital asthma care as a proxy for outcome.

(Quality in Health Care 1997;6:194-198)
\end{abstract}

Keywords: readmission rates; acute asthma; corticosteroids; audit

GGHB Clinical Audit, Dalian House, PO Box 15327, 350 St Vincent Street, Glasgow, UK R Slack, clinical audit facilitator

C E Bucknall, registrar in respiratory medicine

Correspondence to: Mrs R Slack, GGHB Clinical Audit, Dalian House, PO Box 15327, 350 St Vincent Street, Glasgow G3 8YU, UK.

Accepted for publication 5 November 1997 with largely inconclusive results for most medical conditions ${ }^{4-7}$ and with conflicting results in acute asthma. ${ }^{89}$ An initial study showed a posi- tive association between the process of care and readmission, ${ }^{8}$ which was not subsequently confirmed. ${ }^{9}$ That study did not, however, set out to consider this particular issue, and there were also other confounding factors operating. Preliminary results, ${ }^{10}$ recently confirmed, ${ }^{11}$ from a randomised controlled trial that assessed the impact of structured discharge planning in children with acute asthma has shown that the readmission rate declined in the group given more intensive structured discharge planning. If an association between readmission and the preceding process of health care were to be established, readmission rates would be a useful indicator of the quality of health care, and could be accepted as an outcome of health care. ${ }^{12}$ It would also support the audit of process as a proxy for outcome, in acute asthma.

In general, acute severe asthma requires treatment with systemic (oral or intravenous) corticosteroids, given as soon as possible after the diagnosis is made, together with nebulised or high dose inhaled bronchodilators. Recovery can be documented by monitoring peak expiratory flow rates (PEF), with a portable device at the bedside. Patients are usually discharged on a combination of oral and inhaled corticosteroids, with specific review planned, as this is an unstable phase of their illness (box 1).

- Treatment with systemic corticosteroids as soon as possible

- Nebulised or high dose inhaled bronchodilators

- Monitor peak expiratory flow rates

- Discharged on a combination of oral and inhaled corticosteroids

- Specific review planned

Box 1 Standard management of acute asthma.

Information on important aspects of the process of care, based on guidelines, can be collected with a standard form, supplied by the BTS. ${ }^{13}$ These criteria describe the severity of the episode, aspects of hospital care, and discharge procedure. Patient specific readmission rates (readmissions of individual patients to any hospital in Scotland with a specific diagnosis) are available from routinely collected data in Scotland. ${ }^{14}$ This allows for the identification of hospitals with low and high readmission rates.

This study was designed to test the hypothesis that hospitals with significantly different readmission rates for acute asthma would show significantly different degrees of adherence to the recommended audit criteria, in groups of 


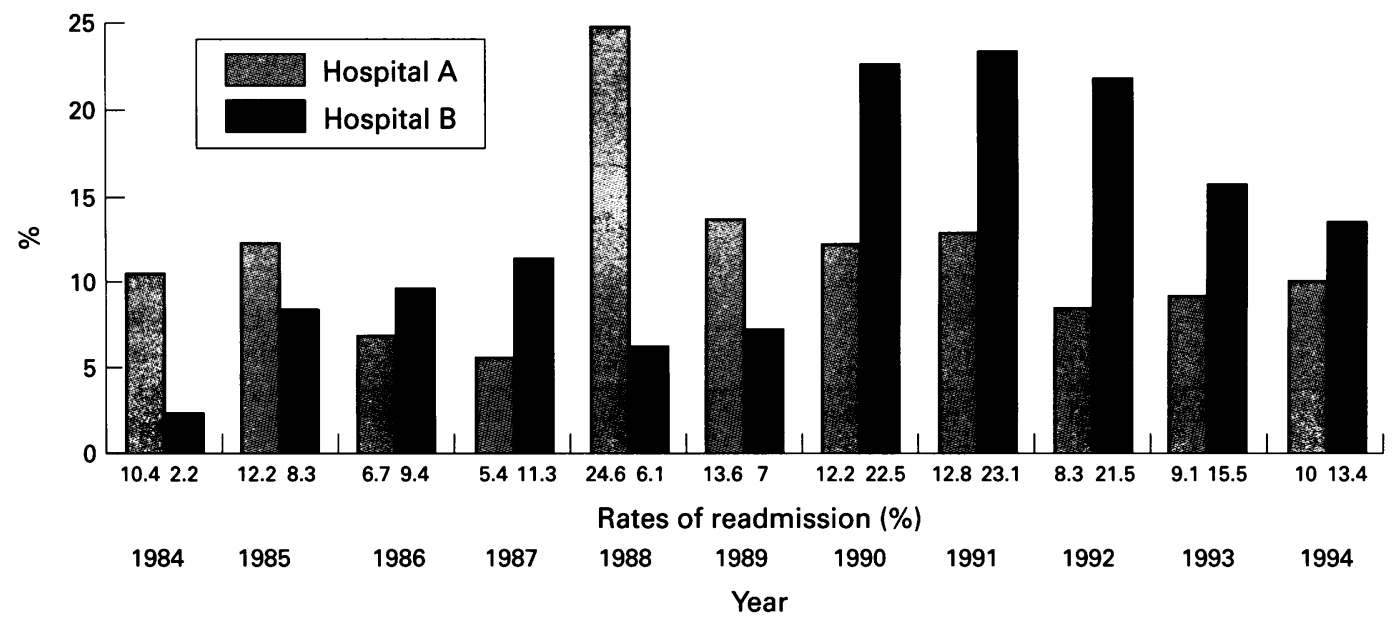

Readmission rates are associated with differences in the process of care in acute asthma.

patients presenting with episodes of similar severity.

\section{Study design}

READMISSION RATES, AND THE IDENTIFICATION OF HOSPITALS WITH LOW AND HIGH READMISSION RATES

A readmission was defined as an admission with acute asthma occurring within two months of a previous admission with acute asthma. The 16-44 year age band was chosen for the study on the basis that the diagnosis of asthma is most secure in this group, with fewer confounding morbidities, or presence of irreversible airways disease. National readmission data on patients discharged with a principle diagnosis of acute asthma (ninth revision of the international classification of diseases (ICD-9) 493) were obtained from the Scottish Office Information and Statistics Division. From this two hospitals were identified as having a "low" (hospital A) and a "higher" (hospital B) readmission rate. The linkage system enabled us to exclude the possibility that a low readmission rate was due to patients being admitted elsewhere. To allow trends over time to be followed, this information was requested for the 10 year period, 1983-93, for the two identified hospitals (figure). Admissions for 1992 were chosen for the study, as rates had been stable in each hospital for some time.

SUBJECTS: SELECTION OF A RANDOM COHORT All 1992 admissions to hospitals A and B were randomised and a sample of 50 was made. At the first sampling, four case records were not found in hospital A and three in hospital B. The next cases in sequence on the randomised list were taken, to complete a sample of 50 from each hospital.

\section{DATA COLLECTION}

Assessing the process of care

The process of care was measured with the criteria recommended by the BTS.

- PEF was recorded on admission-taken from accident and emergency record, or first assessment, if patients were admitted directly to a ward

- Early administration of systemic corticosteroids-administration of intravenous hydrocortisone or oral prednisolone, taken from accident and emergency record, or from the record of initial treatment if taken straight to the ward

- PEF monitoring in the 24 hours before discharge-taken from a peak flow chart. When at least two PEF measurements were available in the 24 hours before discharge PEF variability was calculated

- Whether inhaled corticosteroids were prescribed on discharge, and any change in dose compared with admission-taken from the immediate discharge letter, the discharge summary, or if there were repeated references to this in the clinical notes-for example, when patients had medication available to them at home. When inhaled steroid at discharge was recorded, the dose was further categorised as started, increased, maintained, or reduced compared with the dose being taken before admission

- Whether oral corticosteroids were given at discharge-taken from the immediate discharge letter or full summary

- Follow up arrangements - the nature of planned outpatient review, including clinic specialty, as well as whether patients were advised to see their general practitioner within a week of discharge

- Nature of self management plans discussed before discharge-taken from clinical notes or discharge information.

\section{Assessing possible confounding factors}

Assessing possible confounding factors caused by the groups not being similar in terms of demographic characteristics or severity of the presenting attack:

- PEF on admission-recorded as already described; the lower the PEF the more severe the attack

- Partial pressure of $\mathrm{CO}_{2}$ on admission $\left(\mathrm{pCO}_{2}\right)$-recorded from measurements of arterial blood gases in the accident and emergency record or first assessment in the ward; $\mathrm{CO}_{2}$ is a normal product of respiration: $\mathrm{pCO}_{2}$ commonly falls during an episode of acute wheezing, as patients overcompensate for airflow obstruction by overbreathing to an extent: retention of $\mathrm{CO}_{2}$ is a recognised objective marker of severity ${ }^{15}$ 
Table 1 Patient characteristics

\begin{tabular}{|c|c|c|c|c|}
\hline \multirow[b]{2}{*}{ Characteristic } & \multirow[b]{2}{*}{ Hospital $A$} & \multirow[b]{2}{*}{ Hospital B } & \multicolumn{2}{|l|}{$95 \% C I$} \\
\hline & & & $P_{A} P_{B}$ & $\mu_{A}-\mu_{B}$ \\
\hline Sample size (n) & 50 & 50 & & \\
\hline Female (\%) & 64 & 62 & -18 to 22 & \\
\hline Average age (y) & 30 & 32 & - & -5.5 to 1.7 \\
\hline \multicolumn{5}{|c|}{ Deprivation category: } \\
\hline$\leqslant 5(\%)$ & 22 & 24 & -19 to 15 & - \\
\hline$=6(\%)$ & 22 & 27 & -22 to 12 & - \\
\hline$=7(\%)$ & 56 & 49 & -13 to 27 & - \\
\hline
\end{tabular}

$P_{A}-P_{B}$ Proportion in hospital A minus proportion in hospital $B ; \mu_{A}-\mu_{B}$ mean value in A minus mean value in $B ; \chi^{2}$ for distribution of deprivation categories $=0.38, \mathrm{df}=2, \mathrm{p}=0.8$.

Pulse rate on admission-taken from the accident and emergency record or first assessment in the ward ${ }^{15}$

- Deprivation category, derived from the postcode of residence of the patient, categorised into seven groups, with category 1 indicating the most affluent area of residence and category 7 the most deprived ${ }^{16}:$ - this was collected because of evidence of greater deprivation being associated with higher rates of admission and readmission with acute asthma (see discussion)

- Duration of hospital stay-recorded from routine data; the duration of oral corticosteroids treatment after discharge could be influenced by the duration of treatment in hospital.

All these variables described with the exception of admission pulse rate, duration of hospital stay and deprivation category of area of residence, constitute the recommended BTS dataset for audit of the management of acute asthma care in hospital. These variables (summarised in box 2) were collected from case notes retrospectively by RS.

BTS AUDIT CRITERIA
Episode severity
- Admission PEF
- $\mathrm{pCO}_{2}$
Hospital management
- Early administration of corticosteroids
- PEF recording in 24 hours before dis-
charge
Discharge process
- Inhaled corticosteroids and change in dose
- Oral corticosteroids
- Follow up arrangements
- Self management plan
ADDITIONAL DATA colleCTED
- Admission pulse rate
- Postcode of residence
- Duration of hospital stay

Box 2 Study dataset.

ANALYSIS

Continuous variables were compared with a two sample $t$ test and 95\% confidence interval ( $95 \% \mathrm{CI})$ for the difference in means, or by Mann-Whitney comparison of medians, when data were not normally distributed. Categorical variables were compared with the $\chi^{2}$ test and $95 \%$ CIs for the difference between proportions. The study had $85 \%$ power to detect a $30 \%$ difference in proportions at the $5 \%$ level of significance. ${ }^{17}$

\section{CONFIDENTIALITY}

As the study required the review of identifiable patient information, the local Director of Public Health was approached to endorse the application to the Scottish Office Information and Statistics Division. The medical director in each hospital gave permission to review case records. Assurance was given that only aggregated and anonymised data would be reported.

\section{Results}

The readmission rate for hospital A was $7 / 84$ (8.3\%) and for hospital B 20/91 (21.5\%) for the period October 1991 to September 1992.

In each hospital sample three cases were excluded as there was no evidence of the admission having been for the management of acute asthma (four were for non-acute asthma, one for other medical reasons, and one patient was admitted for bronchoscopy).

\section{POSSIBLE CONFOUNDING VARIABLES \\ Demographic}

Table 1 shows the characteristics of the sample cases. As there were too few patients from deprivation categories 1,2 , and 3 to allow comparison, the more affluent categories were amalgamated into a group with deprivation category $\leqslant 5$. There was no difference in the distribution of patients from more or less deprived areas of residence between the two hospitals.

\section{Duration of stay}

This ranged from 0 to 10 days in hospital $\mathrm{A}$, with a median of three days. Apart from two outliers of 98 and 82 days, the range in hospital B was 0-20 days, with a median of three days (Mann-Whitney comparison of medians $95 \%$ CI B-A 0.0 to 2 ).

\section{Severity of the presenting episode}

Table 2 shows details of the severity of the presenting episode. There were no differences in pulse, PEF, or mean $\mathrm{pCO}_{2}$ at admission. Arterial blood gases were not recorded or were missing in eight $(17 \%)$ cases from hospital $\mathrm{A}$ and $13(28 \%)$ from hospital $B$.

\section{MEASUREMENTS OF PROCESS OF CARE}

\section{Use of corticosteroids on admission}

Hospital A gave systemic corticosteroids on admission to $36 / 45(80 \%)$, whereas hospital B gave them to $26 / 45(58 \%)\left(\chi^{2}=5.184 ; p=0.02\right.$ : $95 \%$ CI A-B $3.4 \%$ to $41 \%$ ).

\section{Use of corticosteroids on discharge}

Similar numbers of patients in each hospital sample, $38 / 47(81 \%)$, were recorded as taking inhaled corticosteroids at discharge. For further analysis, the numbers of patients in whom the dose was maintained or reduced were grouped together and compared with those in whom the dose was started or increased. More patients in hospital A, 23/38 (61\%), had their inhaled 
Table 2 Severity of episode

\begin{tabular}{lllllll}
\hline & Hospital $A$ & & Hospital B & & $95 \%$ CI & p Value \\
\hline & Mean (SD) & $n$ & Mean $(S D)$ & $n$ & Mean $A-B$ & $t$ Test \\
Pulse (bpm) & $108(17)$ & 43 & $104(17.6)$ & 45 & -3.1 to 11.6 & 0.25 \\
pCO $_{2}(\mathrm{kPa})$ & $5.32(1.73)$ & 39 & $4.86(1.70)$ & 34 & -0.34 to 1.27 & 0.25 \\
& Median (SD) & $n$ & Median $(S D)$ & $n$ & Median $A-B$ & Mann-Whitney \\
PEF $(1 / \mathrm{m})$ & $160(124)$ & 37 & $200(73)$ & 38 & -70 to 30 & 0.3 \\
\hline
\end{tabular}

corticosteroids started or the dose increased compared with $12 / 38(32 \%)$ in hospital B ( $\chi^{2}=$ $5.9 ; \mathrm{p}=0.02 ; 95 \%$ CI A-B $10 \%$ to $48 \%$ ).

Of patients from hospital A $44 / 47$ (94\%) were discharged on oral corticosteroids compared with $37 / 47(79 \%)$ from hospital $B\left(\chi^{2}=\right.$ $4.4 ; \mathrm{p}=0.04 ; 95 \%$ CI A-B $2 \%$ to $28 \%$ ). Both hospitals discharged $14 / 47(30 \%)$ of their sample on long term oral corticosteroids or to be continued until review $\left(\chi^{2}=0.0 ; p=1.0\right.$; $95 \%$ CI A-B $-18.5 \%$ to $18.5 \%$ ). The median duration of oral corticosteroid treatment, for those discharged on a short course of treatment, was 12 days $(n=29)$ in hospital $A$ and seven days $(n=23)$ in hospital $B$ (MannWhitney comparison of medians $p=0.02 ; 95 \%$ CI A-B 1 to 8).

\section{Follow up arrangements and self management plans}

Forty one patients from hospital A and 40 from hospital $\mathrm{B}$ had follow up arrangements recorded $\left(\chi^{2}=0.09 ; \mathrm{p}=0.8 ; 95 \%\right.$ CI A-B $-12 \%$ to $16 \%)$. Similar proportions, $36 / 41(88 \%)$ in hospital A and 39/40 (97\%) in hospital B, had follow up arranged at a respiratory clinic (expected numbers were too small for a valid $\chi^{2} ; 95 \%$ CI B-A $-1 \%$ to $21 \%$ ). Information on whether the patients were advised to see their general practitioners and on whether there were written self management plans was not recorded in the case notes of patients in either hospital, with one exception.

\section{PEF before discharge}

Twenty eight cases in hospital A and 22 in hospital $B$ had sufficient data to allow PEF variability before discharge to be calculated. In hospital A 14/28 (50\%) had PEF variability $>25 \%$. In hospital B 7/22 (32\%) cases had $>25 \%$ variability $\left(\chi^{2}=1.67 ; \mathrm{p}=0.2 ; 95 \% \mathrm{CI}\right.$ A-B $-9 \%$ to $45 \%)$.

\section{Discussion}

This study shows a clear difference in the process of care between two hospitals with significantly different readmission rates, showing that better care, as defined and measured, is associated with lower readmission rates. It provides definitive evidence of an association between process and outcome, allowing readmission rates with acute asthma to be accepted as an outcome of health care. ${ }^{12}$

It was important to assess carefully any confounding due to differences in patient populations or severity of the presenting episode. We have shown the groups to be similar demographically. Furthermore, although there was no significant difference detected in the variables used to measure severity of the presenting episode, the trend in all these variables (PEF, pulse rate, and $\mathrm{pCO}_{2}$ at admission) suggested that patients presenting to hospital $\mathrm{A}$ (with the lower readmission rate) were, if anything, of marginally greater severity. The similarity in proportions with notable PEF variability before discharge is another marker for the groups being similar in severity. There was some evidence, ${ }^{18} 19$ more recently refuted by a prospective study designed to test this, ${ }^{20}$ that patients with greater PEF variability before discharge were at greater risk of readmission.

No account could be taken of criteria for referral from primary care, in this retrospective review of case notes. There is some evidence of admission rates for acute asthma being higher in areas of social deprivation ${ }^{21}$ and of readmission rates being associated with greater social deprivation. ${ }^{22}$ Notwithstanding any possible differences in referral or admission policies, the samples were similar for age, sex, severity of the presenting episode, and the deprivation category of the patients' area of residence.

A limitation of the study is the sample size. Although the samples were small, the analysis showed significant differences in the process of care. Missing data could bias results, particularly in a study with a small sample size. This seemed to affect both samples equally-for example, the recording of two PEFs in the 24 hours before discharge. In other cases the variable with many missing data was supported by other measures. Measurement of episode severity could have been biased by fewer recordings of $\mathrm{pCO}_{2}$ in hospital $\mathrm{B}$, but comparisons of pulse and PEF measurements confirmed the similarity of the groups.

It is clearly important to look for the reasons underlying differences in the process of care between the two hospitals. For example, although temporarily increasing medication, including inhaled corticosteroids, is recommended after an episode of acute severe asthma, there may be reasons why this is not done. Patients with severe disease may already be taking high doses of oral and inhaled corticosteroids, leaving little scope for further increases. If this were the explanation for the lesser tendency to increase inhaled corticosteroids in hospital B, it would be expected that more of the patients of that hospital would be discharged on long term oral corticosteroids. This was not found to be the case. Likewise, a longer stay in hospital might affect the duration of time that oral corticosteroids were continued after discharge, but the median duration of stay was similar in the two groups. There can be no doubt that systemic corticosteroids were given sooner after admission and inhaled and oral corticosteroids were used more intensively in the hospital with the lower readmission rate.

The evidence for a link between process and outcome found here is therefore robust.

Published evidence linking readmission with the preceding quality of care is confused. Frankl et al found potentially avoidable lapses in the quality of care of readmitted patients and suggested that stricter assessment before discharge and coordination of follow up might reduce readmission. ${ }^{4}$ Ludke et al, however, found inadequate discharge planning to be 
common both in general medical patients who were and were not readmitted, suggesting that other factors were also operating. ${ }^{6}$ Readmissions in patients with obstructive lung disease were linked to variations in standards of care, but not all readmissions were identified in that study, which also studied only male veterans. In a review, Milne and Clark did not endorse the use of readmission rates as a direct outcome measure for general medical patients. ${ }^{23}$

Improvements in adherence to guidelines on acute asthma over three consecutive audits were shown in one hospital, in which initial audit showed an association between process and outcome, ${ }^{8}$ which was not subsequently confirmed. ${ }^{9}$ In the second study, however, major improvements in the process of care in hospital, but not at the stage of discharge planning were found. Madge et al recently reported reduced readmission rates in association with structured discharge planning. ${ }^{11}$

It is interesting to speculate on possible differences in the organisation of care that may underlie the differences in process and outcome found, but this study did not set out to examine differences in the structure of care. The hospitals were chosen for their different readmission rates, but also because they were considered to handle acute emergencies within a similar framework, as well as both being city centre hospitals with similarly deprived catchment areas. Organisational issues are best considered locally, in the light of process and outcome data, to make the link between observed deficiencies and possible causes, ${ }^{24}$ particularly as the evidence that different organisational frameworks influence outcome in acute asthma is limited to the finding that specialists manage the condition better. ${ }^{85}$

The importance of the finding of a robust association between process and outcome shown here is that it allows for the possibility of routine outcome data, where there is reasonable certainty of their accuracy, to be used to monitor the quality of care with persistently high readmission rates triggering more detailed audit. Sustained higher and lower readmission rates have been investigated in this study. The figure shows that these can vary from year to year, a phenomenon which we have not investigated. The accuracy of readmission data is important. In Scotland, a record linkage system allows readmission to any Scottish hospital to be identified. ${ }^{1+}$ It allowed this study to be based on sound data, which can be requested by individual hospitals or commissioning authorities. Outside Scotland, in areas served by a single district general hospital, local hospital readmission rates may be relatively accurate, allowing their use as an outcome indicator. In cities, where readmission to several hospitals may occur, this approach will not always be valid. Another weakness of using asthma readmission rates to prompt action on quality improvement is the time delay before they become available. Where research has shown an association between process and outcome, audit of process can be justified as a proxy measure of outcome.
This study supports the hypothesis that for acute asthma requiring hospital admission a poorer outcome (higher readmission rates) occurs where recommended practice is implemented less consistently. The audit criteria recommended by the BTS are valid as proxy measures of outcome, as they identified significant differences in the process of care. These findings support the concept of regular audit of process of care in this condition, before the delayed evidence of readmission rates prompts action.

We acknowledge the cooperation and assistance received from the clinicians and medical records staff at the two hospitals and the invaluable assistance of staff at the Information and Statistics Department, Edinburgh, without which this study would not have been possible. RS was funded by the combined National Asthma Campaign and CRAG (Scottish Office) funds to administer a confidential inquiry into asthma deaths in Scotto administer a confidential inquiry into asthma deaths in Scot-
land. The work described in this paper was undertaken as part land. The work described in this paper was undertaken
of a Master of Public Health degree in her own time.

1 British Thoracic Society, Research Unit of the Roval College of Physicians of London, King's Fund Centre, NAC. Guidelines for management of asthma in adults: 11-acute suidelines for management of asthma in

2 British Thoracic Society and others. Statement of the British Thoracic Society on the management of asthma. Thorax 1993;48:s 1-24

3 British Thoracic Society and others. The British guidelines on asthma management 1995 review and position statement. Thorax 1997;52(suppl 1):s1-21.

4 Frankl SE, Breeling JL, Goldman L. Preventability of emergent hospital re-admission. Am f Med 1991;90:667-74

5 Panzer RJ. Hospital re-admissions and quality of care. $A m \mathcal{f}$ Med 1991;90:665 -6.

6 Ludke RL, Booth BM, Lewis-Beck JA. Relationship between early re-admission and hospital quality of care between early re-admission and hos

7 Ashton CM, Kuykendall DH, Johnson ML, et al. The association between the quality of in-patient care and early re-admission. Ann Intern Med 1995;122:415-21.

8 Bucknall CE, Robertson C, Moran F, et al. Differences in hospital asthma management. Lancet 1988;ii:748-50.

9 Bucknall CE, Robertson C, Moran F, et al. Improving management of asthma: closing the loop or progressing along the audit spiral? Quality in Health Care 1992;1:15-20.

10 Madge P, Paton JY. Can nursing intervention improve outcome in children hospitalised with acute asthma? A randomised controlled study [abstract]. Eur Respir $\mathcal{F} 1995$; 8:115.

11 Madge P, McColl J, Paton J. Impact of a nurse-led home management training programme in children admitted to hospital with acute asthma: a randomised controlled study Thorax 1997;52:223-8.

12 Shanks J, Frater A. Health status, outcome, and attributability: is a red rose red in the dark. Quality in Health Care 1993;2:259-62.

13 The British Thoracic Society asthma audit database (hospital management of acute severe asthma) [letter]. Quality in Health Care 1995;4:230

14 Kendrick S, Clarke J. The Scottish record linkage system. Health Bull (Edinb) 1993;51:72-8.

15 Arnold AG, Lane DJ, Zapata E. The speed of onset and severity of acute severe asthma. Br 7 Dis Chest 1982;76: 157-63.

16 McLoone P. Carstairs scores for Scottish postcode sectors from the 1991 census. Glasgow: Public Health Research Unit, Glasgow University, 1991

17 Machin D, Campbell MJ. Statistical tables for the design of clinical trials. Oxford: Blackwell, 1987

18 Pearson MG, Ryland I, Harrison BDW. National audit of acute severe asthma in adults admitted to hospital. Quality in Health Care 1995;4:24-30.

19 Bucknall CE, Robertson C, Moran F, et al. Why uncritical criterion based audit is not enough: analysis of PEF data from a prospective asthma audit [abstract]. Thorax from a prosp $1992 ; 47: 884$.

20 Williams TJ, Spencer J, Fahey T, et al. Timing of discharge from hospital of patients admitted with asthma: a district general hospital experience. $₹$ $R$ Coll Physicians Lond 1994; 28:306-9.

21 Shelley M, Croft P, Chapman S, et al. Is the ratio of inhaled corticosteroid to bronchodilator a good indicator of the quality of asthma prescribing? Cross sectional study linking prescribing data to data on admissions. BMf 1996;313 1124-6.

22 Weissman J S, Stern R S, Epstein A M. The impact of patient socioeconomic status and other social factors on readmission: a prospective study in four Massachusett hospitals. Inquiry 1994;31:163-72.

23 Milne R, Clarke A. Can re-admission rates be used as an outcome indicator?. BMF 1990;301:1139-40.

24 Crombie IK, Davie HTO. The missing link in the audit cycle. Quality in Health Care 1993;2:47-8.

25 Osman J, Ormerod LP, Stableforth DE. Management of acute asthma; a survey of hospital practice and comparison acute asthma, a survey of hospital practice and comparison between thoracic and general physicians in Bir 